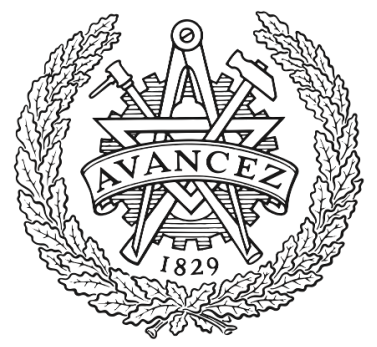

CHALMERS

UNIVERSITY OF TECHNOLOGY

\title{
Selectivity of the First Two Glycerol Dehydrogenation Steps Determined Using Scaling Relationships
}

Downloaded from: https://research.chalmers.se, 2023-04-26 13:58 UTC

Citation for the original published paper (version of record):

Valter, M., dos Santos, E., Pettersson, L. et al (2021). Selectivity of the First Two Glycerol Dehydrogenation Steps Determined Using Scaling

Relationships. ACS Catalysis, 11(6): 3487-3497. http://dx.doi.org/10.1021/acscatal.0c04186

N.B. When citing this work, cite the original published paper. 


\title{
Selectivity of the First Two Glycerol Dehydrogenation Steps Determined Using Scaling Relationships
}

\author{
Mikael Valter,* Egon Campos dos Santos, Lars G. M. Pettersson, and Anders Hellman \\ Cite This: ACS Catal. 2021, 11, 3487-3497 \\ Read Online
}

ABSTRACT: Glycerol is a byproduct of biodiesel production and an abundant feedstock that can be used for the synthesis of highvalue chemicals. There are many approaches for glycerol valorization, but, due to the complicated reaction mechanism, controlling which products are produced is challenging. Here, we describe glycerol's chemical selectivity for different metallic catalysts using descriptors for carbon (mainly $* \mathrm{C}, * \mathrm{CH}_{2} \mathrm{OH}$ ) and oxygen (mainly $* \mathrm{O}, \mathrm{CH}_{3} \mathrm{O} *$ ). The quality of these descriptors and the weighted combinations thereof are validated based on their fit, via linear regression, to the binding energies of all reaction intermediates generated in the first two glycerol dehydrogenation steps on a number of close-packed $\mathrm{Ru}, \mathrm{Co}, \mathrm{Rh}, \mathrm{Ir}, \mathrm{Ni}, \mathrm{Pd}, \mathrm{Pt}, \mathrm{Cu}$,
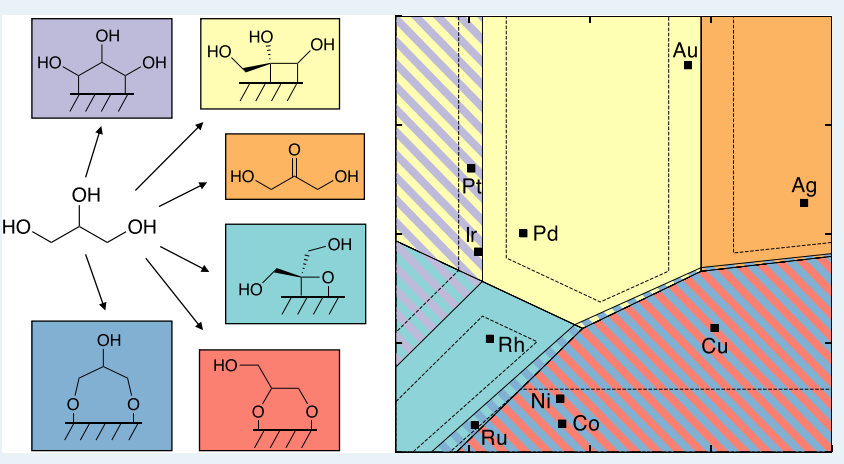
$\mathrm{Ag}$, and $\mathrm{Au}$ surfaces. We show that $* \mathrm{CH}_{2} \mathrm{OH}$ is a better descriptor than ${ }^{*} \mathrm{C}$ for the studied carbon-bound intermediates, which is attributed to the observation that the adjacent $* \mathrm{OH}$ group interacts with the surface. This leads to a negative oxygen dependence, which can be generalized to similar alcohol-derived adsorbates. Furthermore, we show that $\mathrm{CH}_{3} \mathrm{O} *$ is a better oxygen descriptor than $* \mathrm{O}$ for the studied intermediates. This is mainly attributed to the difference between the single and double bonds, as we show that $* \mathrm{OH}$ is closer to the accuracy of $\mathrm{CH}_{3} \mathrm{O}^{*}$. Multilinear regression with different combinations of $* \mathrm{C}, * \mathrm{O}$, and $* \mathrm{OH}$ is comparable in accuracy to that of $* \mathrm{CH}_{2} \mathrm{OH}$ and $\mathrm{CH}_{3} \mathrm{O} *$. Scaling relationships are used to determine the selectivity map for glycerol dehydrogenation. The results show that the first dehydrogenation is selective toward two different intermediates (one bonded via the secondary carbon and the other via the secondary oxygen) depending on the relative bond strength of the carbon and oxygen descriptors. The second dehydrogenation step results in five intermediates, again depending primarily on the relative bond strength of carbon and oxygen to the surface. The selectivity maps can be used together with kinetic considerations and experimental data to find catalyst candidates for glycerol dehydrogenation.

KEYWORDS: scaling, glycerol, $\mathrm{CH}_{2} \mathrm{OH}, \mathrm{CH}_{3} \mathrm{O}$, first principles, selectivity, transition metals

\section{INTRODUCTION}

Over the years, it has become clear that developing renewable and clean energy resources will be essential in the coming decades. In this context, biofuels produced from vegetable oils and waste fats are an alternative to petroleum-based fuels. ${ }^{1,2}$ One concern, due to large-scale production of biofuels, is the massive amount of glycerol that is formed as a byproduct. It is estimated that the biodiesel industry generates hundreds of millions of kilograms of low-value glycerol, ${ }^{3-7}$ and because of its abundance, it is important to find a practical utilization to support the production of biodiesel. One alternative is to use glycerol to produce hydrogen gas for fuel cell applications ${ }^{8-10}$ or to reduce iron ores in steel production. ${ }^{11,12}$ An advantage of glycerol is the fact that the $\mathrm{C}-\mathrm{C}-\mathrm{C}$ backbone is relatively stable, which makes it possible to interrupt the oxidative reaction to form valuable chemical products without producing $\mathrm{CO}_{2}{ }^{4,8}$ Another benefit is that hydrogen generation using glycerol as a starting product can be performed at a lower potential compared to using water and other alcohols. ${ }^{10,13-15}$
In the past few decades, numerous studies have focused on glycerol valorization and how to produce useful products. ${ }^{7,9,10,16}$ One of the methods that has shown promise is glycerol electrooxidation (GEO). ${ }^{17}$ Products of the GEO process include tartronic acid (TA), dihydroxyacetone (DHA), glyceraldehyde (GLYD), glyceric acid (GLYC), hydroxypyruvic acid (HYDP), mesoxalic acid (MESOX), and glycolic acid (GA). All of these are three-carbon products of GEO and they have broad applications. They can be used as food additives, in agriculture, ${ }^{18}$ in detergent production, ${ }^{9}$ in biodegradable polymer synthesis, ${ }^{6}$ as tanning agents in cosmetics, ${ }^{19}$ and in medical applications. ${ }^{20,21}$

Received: September 25, 2020

Revised: February 15, 2021

Published: March 3, 2021 

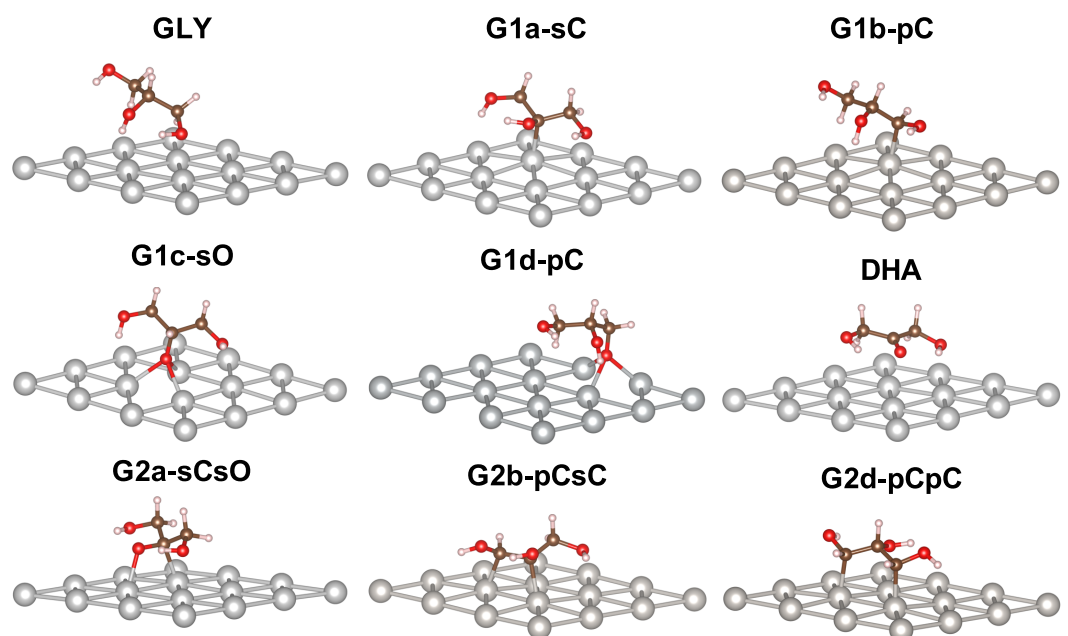

G2b-pCsC
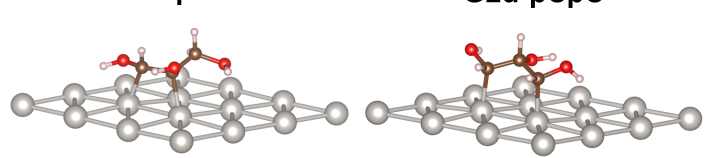

G2i-pOsO

G2j-pOpO
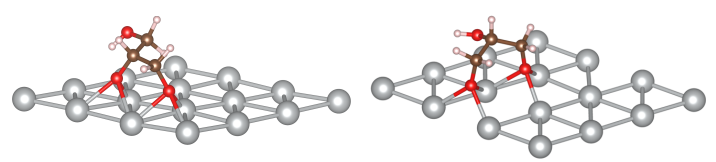

Figure 1. Glycerol intermediate structures. For each intermediate, the optimal adsorption site for most of the metals is shown. The specific adsorption site for each metal is indicated in Table 1 .

As mentioned above, the dehydrogenation of glycerol is a complex chemical process, even when compared with other alcohols. Glycerol is a large molecule, and its possible intermediary steps and products are almost innumerable. ${ }^{8}$ Particularly from an atomistic modeling perspective, it is desirable to determine the energetically preferred species on the surface of the catalyst and find all possible elementary steps that could lead to the products observed experimentally. It involves a series of reaction steps involving $\mathrm{C}-\mathrm{H}$ and $\mathrm{O}-\mathrm{H}$ bond breaking (at higher potentials $\mathrm{C}-\mathrm{C}$ bond breaking is also observed) and, hence, its success depends heavily on the chemical affinity of the catalyst. A material that interacts strongly with an adsorbed carbon atom $\left({ }^{*} \mathrm{C}\right)$ tends to induce more $\mathrm{C}-\mathrm{H}$ bond splitting, while on the other hand, a catalyst that interacts strongly with an oxygen atom $(* \mathrm{O})$ will tend to induce $\mathrm{O}-\mathrm{H}$ bond splitting.

It is known that the adsorption energies for $* \mathrm{O}$ and $* \mathrm{C}$ display linear scaling correlations with the reaction intermediates in many reactions. ${ }^{22-27}$ In this sense, theoretical calculations can be used to classify different materials based on their chemical affinity and thus establish a microscopic description of the reactivity of the catalyst. ${ }^{28,29}$ In addition, it has been found that the activation energy scales linearly with the final-state adsorption energy, that is Brønsted-EvansPolanyi (BEP)-type relationships. ${ }^{26}$ Together, adsorption energy scaling and BEP relationships lead to the concept of volcano curves in catalysis, which is a useful tool to find new catalyst formulations. $^{28,30-32}$

Scaling relations were used by Liu and Greeley ${ }^{33}$ to estimate the binding energies of glycerol intermediates on a number of transition metals based on the binding energy on platinum, assuming that it is proportional to the valency of the adsorbate, as originally proposed by Abild-Pedersen et al. ${ }^{22}$ In comparison to DFT calculations, the thus obtained energies for palladium and rhodium agreed quite well, but larger deviations were reported for copper. This was attributed to the copper surface being unable to break double bonds, in which case the bondcounting valency scheme breaks down. ${ }^{33}$

In the present work, we establish scaling relations for glycerol intermediates with respect to the binding energies of atomic carbon $\left({ }^{*} \mathrm{C}\right)$ and oxygen $\left({ }^{*} \mathrm{O}\right)$ as descriptors without any a priori assumption of valency. In addition, we introduce two new descriptors, hydroxymethyl $\left({ }^{*} \mathrm{CH}_{2} \mathrm{OH}\right)$ and methoxy $\left(\mathrm{CH}_{3} \mathrm{O} *\right)$, which have a better fit to the binding energies of the reaction intermediates and provide a better distinction between different metals. We extensively discuss the reasons for the difference in accuracy of the pairs of descriptors and how this understanding may aid in general predictions of alcohol intermediates. After investigating the sensitivity on the exchange-correlation functional, the two pairs of descriptors are used to generate selectivity maps for the first two steps in glycerol dehydrogenation, which can be used to search for possible catalysts for the reaction.

\section{METHOD}

Density functional calculations were performed using VASP, ${ }^{34-36}$ where the projector augmented wave method ${ }^{37}$ was used to model the interaction between the valence electrons and the core. The Kohn-Sham orbitals were represented using a plane-wave basis set with $450 \mathrm{eV}$ as cutoff energy and a Gaussian smearing of $0.05 \mathrm{eV}$ was applied to the Fermi-level discontinuity. Calculations on $\mathrm{Ni}$ and $\mathrm{Co}$ were performed using spin polarization.

The main exchange-correlation functional applied in the present work, which is used unless otherwise specified, was optB86b-vdW, which includes the non-local effects of electron correlation. $^{38-40}$ optB86b-vdW is known to describe the adsorption of molecules and layered materials accurately. ${ }^{41,42}$ The computational setup used herein was verified in a previous study of methanol adsorption on $\mathrm{Au}^{43}$ Furthermore, the BEEF-vdW functional ${ }^{44}$ was used to determine how sensitive the results are with respect to the exchange-correlation 
Table 1. Optimal Adsorption Sites for Descriptors and Intermediates

\begin{tabular}{|c|c|c|c|c|c|c|c|c|c|c|}
\hline species & $\mathrm{Ag}$ & $\mathrm{Au}$ & Co & $\mathrm{Cu}$ & Ir & $\mathrm{Ni}$ & $\mathrm{Pd}$ & $\mathrm{Pt}$ & $\mathrm{Rh}$ & $\mathrm{Ru}$ \\
\hline $\mathrm{C}$ & $\mathrm{fcc}$ & $\mathrm{fcc}$ & fcc & $\mathrm{fcc}$ & fcc & fcc & fcc & $\mathrm{fcc}$ & fcc & fcc \\
\hline $\mathrm{O}$ & $\mathrm{fcc}$ & $\mathrm{fcc}$ & hcp & $\mathrm{fcc}$ & $\mathrm{fcc}$ & fcc & $\mathrm{fcc}$ & $\mathrm{fcc}$ & fcc & hcp \\
\hline $\mathrm{OH}$ & $\mathrm{fcc}$ & fcc & fcc & $\mathrm{fcc}$ & fcc & fcc & fcc & $\mathrm{fcc}$ & fcc & fcc \\
\hline $\mathrm{CH}_{2} \mathrm{OH}$ & atop & atop & bridge & bridge & atop & bridge & atop & atop & atop & bridge \\
\hline $\mathrm{CH}_{3} \mathrm{O}$ & $\mathrm{fcc}$ & $\mathrm{fcc}$ & $\mathrm{fcc}$ & $\mathrm{fcc}$ & $\mathrm{fcc}$ & fcc & $\mathrm{fcc}$ & $\mathrm{fcc}$ & $\mathrm{fcc}$ & fcc \\
\hline GLY & atop & atop & atop & atop & atop & atop & atop & atop & atop & atop \\
\hline G1a-sC & atop & atop & atop & atop & atop & atop & atop & atop & atop & atop \\
\hline G1b-pC & atop & atop & atop & atop & atop & atop & atop & atop & atop & atop \\
\hline G1c-sO & $\mathrm{fcc}$ & fcc & fcc & fcc & fcc & fcc & $\mathrm{fcc}$ & atop & $\mathrm{fcc}$ & fcc \\
\hline Gld-pO & $\mathrm{fcc}$ & fcc & $\mathrm{fcc}$ & $\mathrm{fcc}$ & atop & $\mathrm{fcc}$ & $\mathrm{fcc}$ & atop & $\mathrm{fcc}$ & fcc \\
\hline DHA & vdW & vdW & & vdW & $\mathrm{vdW}$ & $\mathrm{vdW}$ & vdW & vdW & $\mathrm{vdW}$ & \\
\hline \multirow[t]{2}{*}{$\mathrm{G} 2 \mathrm{a}-\mathrm{sCsO}$} & atop & atop & atop & atop & atop & atop & atop & atop & atop & atop \\
\hline & atop & atop & atop & atop & atop & atop & atop & atop & atop & bridge \\
\hline \multirow[t]{2}{*}{ G2b-pCsC } & $\mathrm{vdW}$ & $\mathrm{vdW}$ & $\mathrm{fcc}$ & vdW & atop & atop & atop & atop & atop & atop \\
\hline & & & atop & & atop & atop & atop & atop & atop & atop \\
\hline \multirow[t]{2}{*}{ G2d-pCpC } & atop & atop & atop & atop & atop & atop & atop & atop & atop & atop \\
\hline & atop & atop & atop & atop & atop & atop & atop & atop & atop & atop \\
\hline \multirow[t]{2}{*}{ G2i-pOsO } & $\mathrm{fcc}$ & bridge & $\mathrm{fcc}$ & $\mathrm{fcc}$ & atop & fcc & $\mathrm{fcc}$ & atop & fcc & fcc \\
\hline & $\mathrm{fcc}$ & atop & $\mathrm{fcc}$ & $\mathrm{fcc}$ & atop & $\mathrm{fcc}$ & bridge & bridge & $\mathrm{fcc}$ & fcc \\
\hline \multirow[t]{2}{*}{ G2j-pOpO } & fcc & fcc & $\mathrm{fcc}$ & $\mathrm{fcc}$ & atop & fcc & fcc & atop & $\mathrm{fcc}$ & fcc \\
\hline & $\mathrm{fcc}$ & $\mathrm{fcc}$ & $\mathrm{fcc}$ & $\mathrm{fcc}$ & atop & $\mathrm{fcc}$ & $\mathrm{fcc}$ & atop & $\mathrm{fcc}$ & $\mathrm{fcc}$ \\
\hline
\end{tabular}

functional. The reason for comparing optB86b/vdWDF and BEEF for the same system is that recent studies indicate that these two functionals are on opposite sides when comparing the over- and under-binding of various molecules, e.g., benzene, naphthalene, on $\mathrm{Pt}^{45,46}$ Finally, the PBE functional ${ }^{47}$ was used for some reference calculations on scaling relations and on van der Waals effects.

The close-packed surfaces-hcp(0001) for Ru and Co, and $\mathrm{fcc}(111)$ for the rest-were modeled as 4-layer $\mathrm{p}(3 \times 3)$ supercells, sampled in a Monkhorst-Pack grid ${ }^{48}$ with $(6 \times 6 \times$ 1) $k$-points. The periodic surface slabs were separated by a vacuum of $20 \AA$. Gas-phase species were computed in a $(20 \times$ $20 \times 20) \AA^{3}$ cell using only the $\Gamma$ point. Structures were relaxed to a maximum residual force of $0.02 \mathrm{eV} / \AA$. The reported adsorbate energies correspond to the optimized adsorption sites, unless otherwise specified.

The binding energy for a species is defined as the energy of the adsorbed species relative to the bare surface and gas-phase reference and the stoichiometric amount of hydrogen gas. The gas-phase reference was glycerol for glycerol derivatives, methanol for $* \mathrm{CH}_{2} \mathrm{OH}$ and $\mathrm{CH}_{3} \mathrm{O} *$, (spin-polarized) oxygen gas for $* \mathrm{O}$, and (spin-polarized) atomic carbon for $* \mathrm{C}$. As the compared structures are similar, in particular with respect to vibrational modes, entropy contributions and zero-point corrections are considered to be small and are not taken into account.

We perform no direct calculations on barriers, neither in a heterogeneous catalysis framework nor in an electrochemical environment. The work is focused on thermodynamics, and barriers are discussed based on previous works.

Scaling relations are established by linear regression, for which the accuracy and reliability are analyzed by calculating 95\% confidence intervals of the slopes, mean absolute errors (MAE), maximum absolute errors (MAX), and coefficient of determination $\left(R^{2}\right)$. It should be noted that MAE and MAX are better error metrics than $R^{2}$ in this work, as $R^{2}$ lacks robustness for small sample sizes, ${ }^{49}$ which is why the analysis is mostly based on MAE and MAX along with the confidence interval.

\section{RESULTS AND DISCUSSION}

In a recent study, ${ }^{50}$ the structure and energy were calculated for all reaction intermediates in glycerol oxidation after the first two deprotonation steps on the close-packed surfaces of cobalt, nickel, copper, ruthenium, rhodium, palladium, silver, iridium, platinum, and gold using DFT. The most stable intermediates and their structure can be seen in Figure 1. For each intermediate, the optimal adsorption site for most metals is shown; the specific adsorption for each metal is indicated in Table 1. Each intermediate is labeled according to the origin (glycerol; G), the number of deprotonations $(0,1,2)$, and an additional running index $(a-j)$ to distinguish intermediates, followed by the carbon and oxygen atoms formally binding to the surface $(\mathrm{p}=$ primary, $\mathrm{s}=$ secondary $)$. Glycerol and dihydroxyacetone are only denoted with abbreviations (GLY and DHA), as they form no formal bonds with the surface but rather interact in a van der Waals fashion. Furthermore, for G2b-pCsC, three bonding mechanisms can be distinguished: The coinage metals do not break the double bond ( $\mathrm{C}-\mathrm{C}$ bond length $\sim 1.35 \AA$ ); $\mathrm{Co}, \mathrm{Ni}$, and $\mathrm{Ru}$ partially break the double bond ( $\mathrm{C}-\mathrm{C}$ bond length $\sim 1.45 \AA$ ) by binding the carbon atoms to one surface atom; $\mathrm{Pd}, \mathrm{Pt}, \mathrm{Rh}$, and Ir break the double bond ( $\mathrm{C}-\mathrm{C}$ bond length $\gtrsim 1.5 \AA$ ) by binding the carbon atoms to separate surface atoms.

Establishing Scaling Relations for Glycerol Intermediates. Using linear regression, we start by fitting the traditional descriptors, the binding energy of carbon $(* \mathrm{C})$ and oxygen $(* \mathrm{O})$, bound at optimal hollow sites, to the computed binding energy of the intermediates as shown in Figure 2a-c, under the assumption that the bond is either carbon-like or oxygen-like (with the exception of G2a-sCsO that has formally one bond of each kind). Since ${ }^{*} \mathrm{C}$ has a valency of four and ${ }^{*} \mathrm{O}$ a valency of two, we expect single carbon-surface bonds to contribute a slope of 0.25 and single oxygen-surface bonds to 

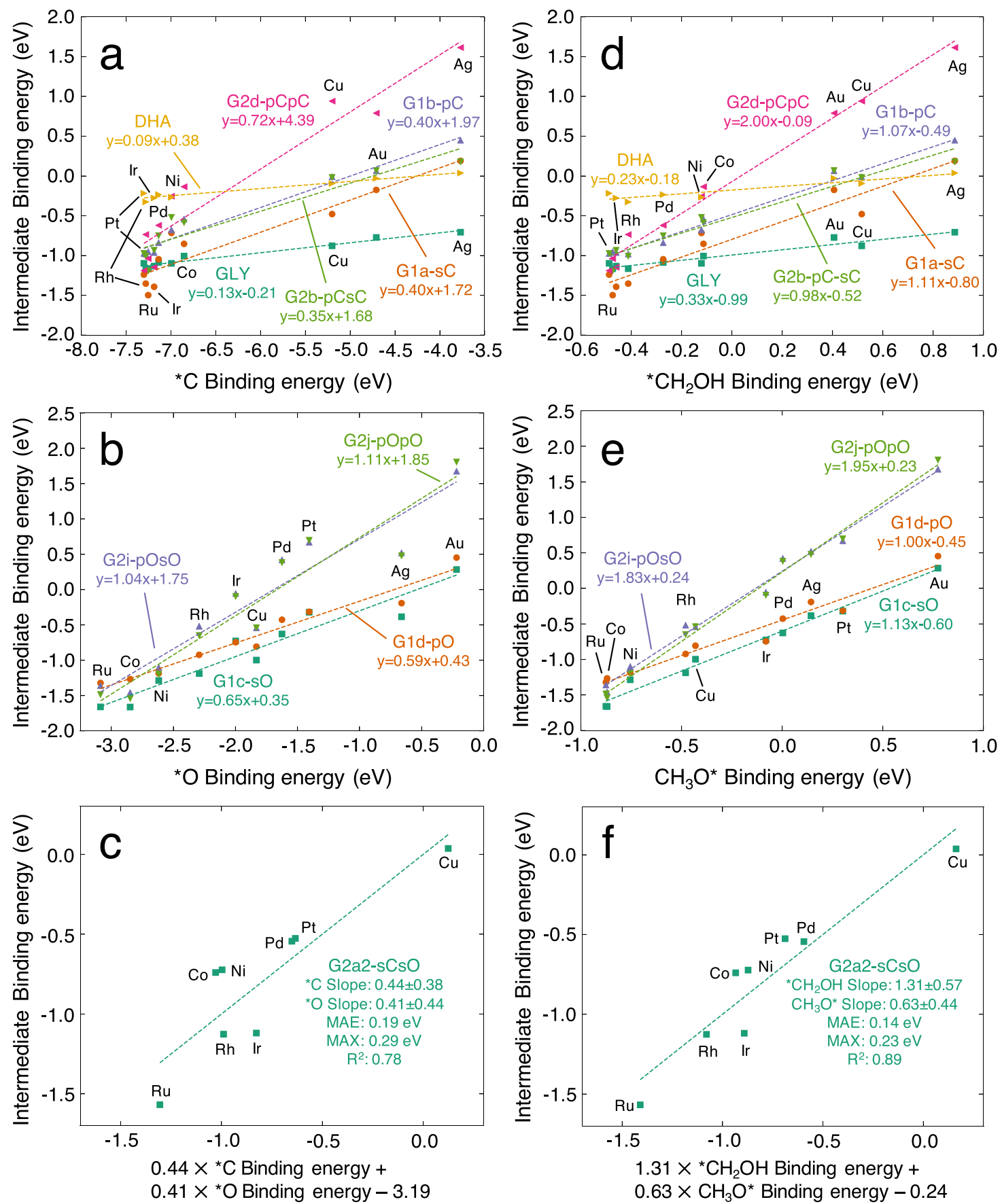

Figure 2. Binding energy $(\mathrm{eV})$ of the relevant reaction intermediates in glycerol oxidation after one and two dehydrogenation steps as a function of ${ }^{*} \mathrm{C}$ and $* \mathrm{O}(\mathrm{a}-\mathrm{c})$ and of ${ }^{*} \mathrm{CH}_{2} \mathrm{OH}$ and $\mathrm{CH}_{3} \mathrm{O} *(\mathrm{~d}-\mathrm{f})$. The data points, trend line, and inset text (showing the reaction intermediate, linear equation, and error metrics where space allows) are color-coded for each intermediate. Confidence intervals and errors may be found in Tables 2 and 3.

Table 2. $* \mathrm{C}$ and $* \mathrm{CH}_{2} \mathrm{OH}$ Scaling Relations ${ }^{a}$

\begin{tabular}{|c|c|c|c|c|c|c|c|c|}
\hline intermediate & ${ }^{*}$ C slope & $\operatorname{MAE}(\mathrm{eV})$ & $\operatorname{MAX}(\mathrm{eV})$ & $R^{2}$ & ${ }^{*} \mathrm{CH}_{2} \mathrm{OH}$ Slope & $\operatorname{MAE}(\mathrm{eV})$ & $\operatorname{MAX}(\mathrm{eV})$ & $R^{2}$ \\
\hline GLY & $0.13 \pm 0.02$ & 0.03 & 0.06 & 0.96 & $0.33 \pm 0.08$ & 0.04 & 0.09 & 0.92 \\
\hline Gla-sC & $0.40 \pm 0.12$ & 0.14 & 0.39 & 0.87 & $1.11 \pm 0.25$ & 0.12 & 0.26 & 0.93 \\
\hline Glb-pC & $0.40 \pm 0.06$ & 0.08 & 0.19 & 0.97 & $1.07 \pm 0.11$ & 0.05 & 0.12 & 0.98 \\
\hline G2b-pCsC & $0.35 \pm 0.11$ & 0.15 & 0.31 & 0.87 & $0.98 \pm 0.20$ & 0.09 & 0.21 & 0.94 \\
\hline G2d-pCpC & $0.72 \pm 0.18$ & 0.25 & 0.39 & 0.91 & $2.00 \pm 0.19$ & 0.09 & 0.16 & 0.99 \\
\hline DHA & $0.09 \pm 0.02$ & 0.02 & 0.06 & 0.94 & $0.23 \pm 0.09$ & 0.04 & 0.07 & 0.86 \\
\hline
\end{tabular}

${ }^{a}$ Confidence intervals and error estimates. 
Table 3. *O and $\mathrm{CH}_{3} \mathrm{O} *$ Scaling Relations ${ }^{a}$

$\begin{array}{ccccc}\text { intermediate } & * \mathrm{O} \text { slope } & \text { MAE }(\mathrm{eV}) & \mathrm{MAX}(\mathrm{eV}) & R^{2} \\ \mathrm{G} 1 \mathrm{c}-\mathrm{sO} & 0.65 \pm 0.15 & 0.14 & 0.30 & 0.92 \\ \mathrm{G} 1 \mathrm{~d}-\mathrm{pO} & 0.59 \pm 0.11 & 0.09 & 0.23 & 0.95 \\ \mathrm{G} 2 \mathrm{i}-\mathrm{pO} \mathrm{sO} & 1.04 \pm 0.28 & 0.27 & 0.55 & 0.90 \\ \mathrm{G} 2 \mathrm{j}-\mathrm{pOpO} & 1.11 \pm 0.30 & 0.27 & 0.63 & 0.90 \\ { }^{a} \text { Confidence intervals and error estimates. } & & \end{array}$

contribute $0.5 .^{22}$ Confidence intervals on the slopes along with other error estimates are displayed in Tables 2 and 3.

As seen in Figure 2a and Table 2, glycerol and DHA have small but clearly positive slopes, which is surprising as these species have no formal surface bond. With the mean and maximum absolute errors being less than or equal to 0.03 and $0.06 \mathrm{eV}$, respectively, and the coefficient of determination being 0.94 or higher, the relations are quite accurate. Moving on to the formally carbon-bound species, we expect G1a-sC and G1b-pC to have one carbon bond and G2d-pCpC to have two carbon bonds, while $\mathrm{G} 2 \mathrm{~b}-\mathrm{pCsC}$ could have anything between zero and two based on whether the $\mathrm{C}-\mathrm{C}$ double bond is broken or not, as discussed above. The slope for both G1a-sC and G1b-pC is 0.40 and that for G2d 0.72; these are all greater than the formally expected 0.25 and 0.5 , which do not fall within the confidence intervals. G2b-pCsC has a slope of $0.35 \pm 0.11$, which suggests a single bond, based both on the results for G1a-sC and G1b-pC and on the fact that 0.25 falls within the interval. The errors are relatively large, in particular for G1a-sC and G2d-pCpC, with MAE being 0.14 and $0.25 \mathrm{eV}$, respectively, and MAX being almost $0.4 \mathrm{eV}$. There is an agglomeration of $* \mathrm{C}$ binding energies for the non-coinage metals and a large separation for the coinage metals. While this behavior is in line with the literature, ${ }^{2,51}$ the predictive power is low as it is hard to distinguish between the non-coinage metals. In the case of G1a-sC and $\mathrm{G} 2 \mathrm{~d}-\mathrm{pCpC}$, in particular, there is a steeper trend among these metals reflected in the large maximum absolute errors.

For the singly oxygen-bonded intermediates (Figure $2 \mathrm{~b}$ and Table 3), we get a slope of $0.65 \pm 0.15$ and $0.59 \pm 0.11$ for $\mathrm{G} 1 \mathrm{c}-\mathrm{sO}$ and $\mathrm{G} 1 \mathrm{~d}-\mathrm{pO}$, respectively, with the expected 0.5 from a single oxygen bond at the lower ends of the intervals. The values for the intermediates with two formal bonds, G2i and G2j $(1.04 \pm 0.28$ and $1.11 \pm 0.30)$, have the expected 1.0 well within the intervals. However, the errors are also in this case large, in particular for G2i-pOsO and G2j-pOpO (MAE of 0.27 $\mathrm{eV}$ and MAX of 0.55 and $0.63 \mathrm{eV}$ ). The reason for this can be seen in Figure $2 b$, as copper and silver follow a trend below the fitted line, while platinum, palladium, and iridium follow another trend above it.

Through multilinear regression, $\mathrm{G} 2 \mathrm{a}-\mathrm{sCsO}$ is fitted to a weighted combination of $* \mathrm{C}$ and $* \mathrm{O}$ (Figure $2 \mathrm{c}$ ). G2a-sCsO binds through one carbon and one oxygen, which formally should give coefficients 0.25 and 0.5 for $* \mathrm{C}$ and $* \mathrm{O}$, respectively. As we find the coefficients to be $0.44 \pm 0.38$ and $0.41 \pm 0.44$, with an MAE of $0.19 \mathrm{eV}, \operatorname{MAX}$ of $0.29 \mathrm{eV}$, and $R^{2}$ of 0.78 , it is clear that the fit is not very accurate. At most, it can be said that it appears as though the carbon bond contributes more than the oxygen bond in this representation.

To investigate the issues with the fits to $* \mathrm{C}$ and $* \mathrm{O}$, we fit the binding energies to two alternative descriptors, $* \mathrm{CH}_{2} \mathrm{OH}$ and $\mathrm{CH}_{3} \mathrm{O}^{*}$ (Figure $2 \mathrm{~d}-\mathrm{f}$ and Tables 2 and 3 ), based on the assumption that the similar alcohol structure will make them more similar to glycerol intermediates, with a better balance between carbon-like and oxygen-like bonds. Since these descriptors can each form one formal bond, the slopes should simply be the number of bonds of the relevant intermediate (which can form more than one bond) according to the valency model. The calculated slopes for the singly deprotonated species are in the range 1.00-1.13, with the expected 1.0 within or just outside the confidence interval, while $\mathrm{G} 2 \mathrm{~d}$-pCpC, G2i-pOsO, and $\mathrm{G} 2 \mathrm{j}$-pOpO are in the range 1.83-2.00, with the expected 2.0 within or just outside the confidence interval. G2b-pCsC has a slope of $0.98 \pm 0.20$, which we again interpret as a single bond. For G2a-sCsO, the expected slope is 1.0 for each descriptor, while the calculated values are $1.31 \pm 0.57$ and $0.63 \pm 0.44$ for $* \mathrm{CH}_{2} \mathrm{OH}$ and $\mathrm{CH}_{3} \mathrm{O} *$, respectively; compared to $* \mathrm{C}$ and $* \mathrm{O}$, the errors are lower across the board, with the exception of GLY and DHA. Furthermore, there is a greater spread in terms of descriptor binding energies ( $x$-axis) when $* \mathrm{CH}_{2} \mathrm{OH}$ is used as a descriptor. The latter aspect allows more robust discrimination among the non-coinage metals.

Descriptor Analysis. Why are $* \mathrm{CH}_{2} \mathrm{OH}$ and $\mathrm{CH}_{3} \mathrm{O} *$ better descriptors for glycerol intermediate binding energies than $* \mathrm{O}$ and $* \mathrm{C}$ ? One thing to test is the effect of the matching adsorption sites, as Abild-Pedersen et al. ${ }^{22}$ showed that matching the adsorption site improves the scaling relations. Since the goal of the present work is to predict the optimal binding energy of glycerol intermediates, without knowing the optimal adsorption site on each metal (or alloy) surface a priori, the only degree of freedom in this regard is to fix the adsorption site of the descriptor. Another possible test is to use a descriptor with a single bond, similar to the intermediates and to $\mathrm{CH}_{3} \mathrm{O}^{*}$.

In Table 4, we fit $\mathrm{CH}_{3} \mathrm{O} *$ to $* \mathrm{O}$ (in an optimized adsorption site), $* \mathrm{O}$ atop, and $* \mathrm{OH}$ (in an optimized adsorption site). It

Table 4. Scaling Relations with $\mathrm{CH}_{3} \mathrm{O}^{* a}$

\begin{tabular}{ccccc} 
O descriptor & slope vs $\mathrm{CH}_{3} \mathrm{O} *$ & $\mathrm{MAE}(\mathrm{eV})$ & $\mathrm{MAX}(\mathrm{eV})$ & $R^{2}$ \\
${ }^{*} \mathrm{O}$ & $0.56 \pm 0.16$ & 0.14 & 0.30 & 0.89 \\
${ }^{*} \mathrm{O}($ atop $)$ & $0.54 \pm 0.25$ & 0.21 & 0.49 & 0.75 \\
${ }^{*} \mathrm{OH}$ & $0.99 \pm 0.10$ & 0.06 & 0.14 & 0.98 \\
\multicolumn{2}{l}{${ }^{a}$ Confidence intervals and error estimates. } & & \\
\hline
\end{tabular}

turns out that $* \mathrm{OH}$ is the best fit to $\mathrm{CH}_{3} \mathrm{O} *$. We conclude then that having an oxygen descriptor with one surface bond is the most important in this case. If $* \mathrm{OH}$ is used as a descriptor for the oxygen-bound intermediates, it still is not as accurate as $\mathrm{CH}_{3} \mathrm{O}^{*}$ (see Supporting Information). This can possibly be attributed to the hyperconjugation in the $\mathrm{C}-\mathrm{O}$ bond. ${ }^{52}$

All the carbon-bound intermediates prefer binding atop, while $* \mathrm{CH}_{2} \mathrm{OH}$ binds atop on most metals and in a bridge position on others. In order to investigate the importance of having similar adsorption sites, we fit the intermediates to *C atop in Figure 3 and Table 5. It can be noted that the *C binding energies are more spread out for the non-coinage 
metals, but the errors are comparable to those of the optimized *C (Table 2).

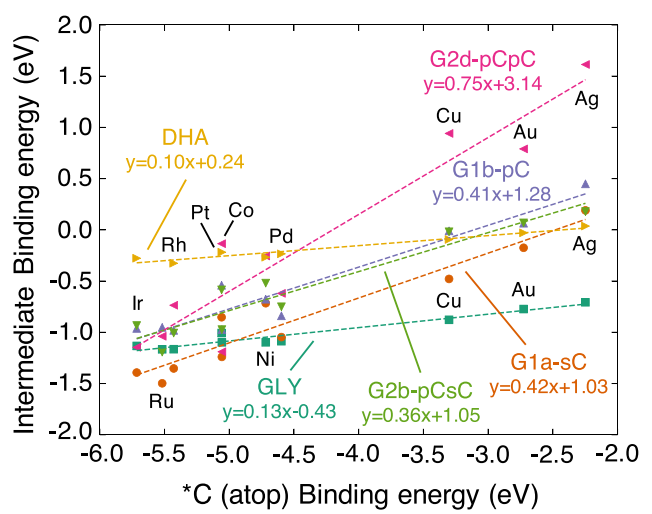

Figure 3. Binding energy $(\mathrm{eV})$ of the relevant reaction intermediates in glycerol oxidation after one and two dehydrogenation steps as a function of $* \mathrm{C}$ atop. The data points, trend line, and inset text (showing the reaction intermediate and linear equation) are colorcoded for each intermediate. Confidence intervals and errors can be found in Table 5 . In comparison to Figure $2 \mathrm{a}$, the ${ }^{*} \mathrm{C}$ binding energies are no longer clustered for the non-coinage metals.

Table 5. *C(atop) Scaling Relations ${ }^{a}$

$\begin{array}{lcccc}\text { intermediate } & * \text { C(atop) slope } & \text { MAE } & \text { MAX } & R^{2} \\ { }^{*} \mathrm{CH}_{2} \mathrm{OH} & 0.38 \pm 0.11 & 0.12 & 0.20 & 0.91 \\ \text { GLY } & 0.13 \pm 0.03 & 0.04 & 0.09 & 0.93 \\ \text { G1a-sC } & 0.42 \pm 0.11 & 0.13 & 0.24 & 0.92 \\ \text { G1b-pC } & 0.41 \pm 0.11 & 0.12 & 0.26 & 0.92 \\ \text { G2b-pCsC } & 0.36 \pm 0.10 & 0.11 & 0.20 & 0.92 \\ \text { G2d-pCpC } & 0.75 \pm 0.24 & 0.27 & 0.56 & 0.88 \\ \text { DHA } & 0.10 \pm 0.02 & 0.03 & 0.04 & 0.94\end{array}$

${ }^{a}$ Confidence intervals and error estimates.

Similar to the oxygen case, we tested to establish a scaling relation between ${ }^{*} \mathrm{CH}_{3}$ and $* \mathrm{CH}_{2} \mathrm{OH}$ as they both have one formal surface bond. With a slope of $1.17 \pm 0.42$, an MAE of $0.16 \mathrm{eV}$, and a MAX of $0.26 \mathrm{eV}$, the fit is, however, not more precise. With $* \mathrm{CH}_{3}$ atop, the slope is $1.37 \pm 0.29$, MAE 0.10 $\mathrm{eV}$, and MAX $0.22 \mathrm{eV}$, which is comparable to the values for ${ }^{*} \mathrm{C}$ in an optimal adsorption site. It can be noted that the slope should be 1.0, which further indicates that something else is missing.

Since neither scaling $* \mathrm{C}$ atop with the atop-bound intermediates nor having the carbon descriptor with a single bond, like the intermediates, resolves the discrepancy between ${ }^{*} \mathrm{C}$ and $* \mathrm{CH}_{2} \mathrm{OH}$, we investigate whether the oxygen also interacts with the surface. Such interaction is indicated by the fact that among the non-coinage metals, the oxygen-surface distance varies from $2.25 \AA$ on ruthenium to $3.15 \AA$ on platinum, two metals with the same $* \mathrm{C}$ binding energy but very different $* \mathrm{O}$ binding energy. Thus, the intermediate surface bonds would not be described as merely either carbonlike or oxygen-like.

The multilinear regression analysis of $* \mathrm{CH}_{2} \mathrm{OH}$ is shown in Table 6. It can be seen that the dependence on the oxygen descriptor tends to be slightly negative. In the case of the pairs $* \mathrm{C}$, $* \mathrm{O}$ and $* \mathrm{C}, * \mathrm{OH}$, an oxygen-associated slope of zero is within or at the edge of the confidence interval; in isolation, these two results would not imply a statistically significant correlation with a negative slope. However, with ${ }^{*} \mathrm{C}$ atop, the slope of the oxygen descriptor is more clearly negative; this shows that $* \mathrm{C}$ atop is a more accurate descriptor for the carbon contribution of the bond. The multilinear analysis was replicated with the PBE exchange-correlation functional (see Supporting Information) with similar results on $* \mathrm{C}$. Furthermore, ${ }^{*} \mathrm{OH}$ and $\mathrm{CH}_{3} \mathrm{O} *$, i.e., the more accurate oxygen descriptors based on the results presented above, have clearer negative oxygen-associated slopes. These results imply that the quality of the descriptors is important to observe the negative oxygen dependence.

The negative oxygen dependence can be tentatively explained based on the oxygen electronegativity; if the surface interacts weakly with oxygen, charge is taken from the carbon atom, resulting in a less satisfied valency and, as a consequence, stronger carbon interaction with the surface to compensate. On the other hand, if the surface interacts strongly with the oxygen, the metal will provide the charge directly; thus, the carbon has less to gain by interacting with the surface and binds more weakly. Supporting this is the observation that the Bader charge on the carbon atom is $\sim 0.3 e$ lower on $\mathrm{Pt}, \mathrm{Pd}, \mathrm{Ag}$, and $\mathrm{Au}$ with relatively weak oxygen-surface interaction compared with $\mathrm{Co}, \mathrm{Ni}, \mathrm{Cu}$, and $\mathrm{Ru}$ with stronger oxygen preference, while $\mathrm{Rh}$ and $\mathrm{Ir}$ fall halfway between. It can be noted that $\mathrm{Co}, \mathrm{Ni}, \mathrm{Cu}$, and $\mathrm{Ru}$ bind ${ }^{*} \mathrm{CH}_{2} \mathrm{OH}$ on a bridge site, such that a $\mathrm{C}-\mathrm{H}$ bond is stretched, which has been observed for similar systems as a stabilizing donation-backdonation mechanism. ${ }^{53,54}$ However, if only atop configurations (preferred for the rest of the metals) are compared, the charge difference persists, though smaller $(\sim 0.2 e)$. The $\mathrm{C}-\mathrm{O}$ bond length is more stretched for the oxygen-preferring group compared with the carbon-preferring one; as an example, on platinum the bond length is $1.39 \AA$, while on cobalt it is elongated to $1.47 \AA$, because in the former case the bond is more polar covalent. Finally, a fixed carbon affinity but increasing oxygen affinity results in weaker adsorption; cobalt and platinum have the same carbon binding energy atop while all carbon-bound intermediates except GLY and DHA bind stronger on platinum; in particular, it can be seen in Figure 3, e.g., that $\mathrm{G} 2 \mathrm{~d}-\mathrm{pCpC}$ binds $1.0 \mathrm{eV}$ stronger on platinum.

Turning to GLY and DHA, we have seen above that they scale well with $* \mathrm{C}$ and $* \mathrm{CH}_{2} \mathrm{OH}$. As they visually seem to

Table 6. Scaling Relations with $* \mathrm{CH}_{2} \mathrm{OH}^{a}$

\begin{tabular}{|c|c|c|c|c|c|c|}
\hline C descriptor & slope vs $* \mathrm{CH}_{2} \mathrm{OH}$ & O descriptor & slope vs $* \mathrm{CH}_{2} \mathrm{OH}$ & MAE (eV) & $\operatorname{MAX}(\mathrm{eV})$ & $R^{2}$ \\
\hline$* \mathrm{C}$ & $0.41 \pm 0.09$ & $* \mathrm{O}$ & $-0.10 \pm 0.13$ & 0.07 & 0.18 & 0.96 \\
\hline$* \mathrm{C}$ & $0.39 \pm 0.06$ & $* \mathrm{OH}$ & $-0.15 \pm 0.15$ & 0.07 & 0.17 & 0.99 \\
\hline$* \mathrm{C}($ atop $)$ & $0.52 \pm 0.10$ & $* \mathrm{O}($ atop $)$ & $-0.24 \pm 0.15$ & 0.06 & 0.15 & 0.97 \\
\hline *C(atop) & $0.42 \pm 0.07$ & $* \mathrm{OH}$ & $-0.22 \pm 0.16$ & 0.07 & 0.18 & 0.97 \\
\hline *C(atop) & $0.43 \pm 0.08$ & $\mathrm{CH}_{3} \mathrm{O}^{*}$ & $-0.23 \pm 0.17$ & 0.07 & 0.18 & 0.97 \\
\hline
\end{tabular}

${ }^{a}$ Confidence intervals and error estimates. 
interact with a surface atom through their primary $\mathrm{OH}$ group (see Figure 1), it might be worthwhile to investigate their scaling with oxygen descriptors atop. ${ }^{*} \mathrm{O}$ atop vs GLY has a slope of $0.15 \pm 0.10$, an MAE of $0.08 \mathrm{eV}$, and a MAX of 0.15 $\mathrm{eV}$, while DHA has a slope of $0.15 \pm 0.08$, an MAE of $0.05 \mathrm{eV}$, and a MAX of $0.08 \mathrm{eV}$. $\mathrm{CH}_{3} \mathrm{O}^{*}$ atop vs GLY has a slope of $0.33 \pm 0.17$, an MAE of $0.07 \mathrm{eV}$, and a MAX of $0.12 \mathrm{eV}$, while DHA has a slope of $0.30 \pm 0.15$, an MAE of $0.05 \mathrm{eV}$, and a MAX of $0.08 \mathrm{eV}$. So, while the margins of the error are larger and the errors are higher than shown in Table 2, the positive slopes are statistically significant. Similar results have been reproduced using the PBE exchange functional, which lacks $\mathrm{vdW}$ interaction (see Supporting Information). Thus, some caution should be taken in concluding the correlation to be carbon-like. On the other hand, $* \mathrm{C}$ seems to be better at representing the interaction, which can be shown by fitting GLY to $* \mathrm{C}$ and $* \mathrm{O}$ atop, with the clearly positive $* \mathrm{C}$ slope of $0.11 \pm 0.03$ and unclear $* \mathrm{O}$ (atop) slope of $0.02 \pm 0.03$.

$* \mathrm{CH}_{2} \mathrm{OH}$ and $\mathrm{CH}_{3} \mathrm{O} *$ are the most accurate descriptors for all non-vdW intermediates. By multilinear regression, it can be shown that only one of them at a time scales with a given intermediate, with a slope of 0 for the other descriptor within the confidence interval, in addition to not improving the MAE (see Supporting Information). The multilinear regression of combinations of $* \mathrm{C}, * \mathrm{O}$, and $* \mathrm{OH}$ in different adsoption sites shows that the carbon-binding intermediates have a negative dependence on ${ }^{*} \mathrm{OH}$, which in most cases is non-significant due to the relatively large margins of error (see Supporting Information). It is still reasonable to believe that the oxygen dependence for $* \mathrm{CH}_{2} \mathrm{OH}$ plays some role in these intermediates based on the adsorption geometry and the fits to $* \mathrm{CH}_{2} \mathrm{OH}$. The advantage of the multilinear regression of $* \mathrm{C}$, $* \mathrm{O}$, and $* \mathrm{OH}$ is that there are extensive databases on these descriptors, ${ }^{55}$ which can be used to extrapolate to other surfaces.

Exchange-Correlation Functional Sensitivity. It has been shown that the use of optB86b-vdW gives good agreement with the experiment regarding adsorption on coinage metals ${ }^{41,43}$ and, through explicit inclusion of $\mathrm{vdW}$, the interaction between layered materials. ${ }^{42}$ However, it is still important to estimate the sensitivity of the present results to the choice of the functional. In a recent study of the molecular adsorption on $\mathrm{Pt},{ }^{46}$ optB86b-vdW and BEEF-vdW were often found to overbind and underbind, respectively, when compared to experimental data. Here, the BEEF-vdW functional was used to evaluate the sensitivity of our results. Figure 4 shows $* \mathrm{CH}_{2} \mathrm{OH}$ and $\mathrm{CH}_{3} \mathrm{O} *$ evaluated with optB86b-vdW and BEEF-vdW. In terms of slope, the descriptors have almost one-to-one correlation (slope of 0.98 \pm 0.23 and $0.92 \pm 0.20$, respectively) with MAE being 0.08 and $0.05 \mathrm{eV}$ and MAX being 0.14 and $0.09 \mathrm{eV}$. BEEF-vdW underbinds both descriptors relative to optB86b-vdW ( 0.4 and $0.3 \mathrm{eV}$ for $* \mathrm{CH}_{2} \mathrm{OH}$ and $\mathrm{CH}_{3} \mathrm{O} *$, respectively). By this, we conclude that the differences in terms of slope are comparable to the errors in the regression analysis and, consequently, that the choice of xc-functional should not matter for the conclusions regarding the descriptor analysis above.

Selectivity Maps. Based on the simple scaling relations established for the paired descriptors $* \mathrm{C}$, $* \mathrm{O}$ and $* \mathrm{CH}_{2} \mathrm{OH}$, $\mathrm{CH}_{3} \mathrm{O} *$ in Figure 2, we construct thermodynamic selectivity maps for the products of the first and second dehydrogenation steps of glycerol oxidation, which are shown in Figure 5. It should be pointed out that the borders between the regions are

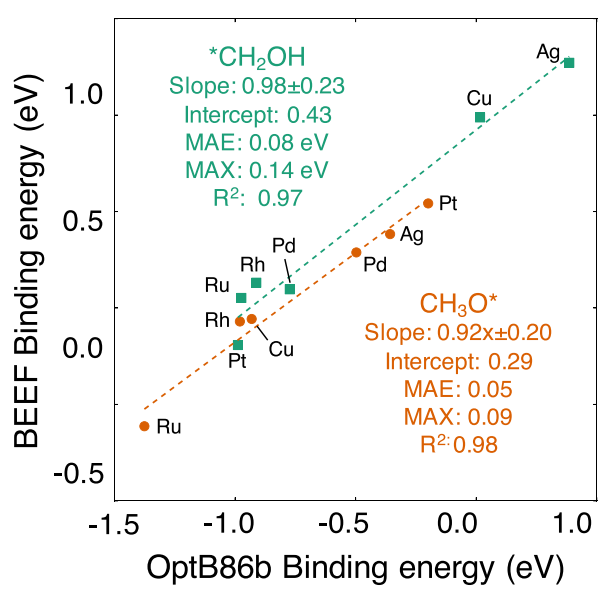

Figure 4. Binding energies of the first dehydrogenation step, evaluated using two different exchange-correlation functionals, optB86b-vdW and BEEF-vdw, both including non-local correlation. Energies are in $\mathrm{eV}$.

not sharp; the selectivity does not go from $100 \%$ product A to $100 \%$ product $\mathrm{B}$ upon crossing. Close to the borders, we assume that there is a Boltzmann-distributed mixture of $\mathrm{A}$ and $\mathrm{B}$ as a function of the energy difference $\Delta E=\left|E_{\mathrm{A}}-E_{\mathrm{B}}\right|$. Thus, the energy margins are shown as dashed lines to indicate sensitivity. The lines are arbitrarily drawn at $\Delta E=0.1 \mathrm{eV}$ difference for clarity, but due to linearity, it can be easily visually extrapolated to other margins.

For the first dehydrogenation step (Figure 5a,5c), both pairs of descriptors show that the secondary-carbon-bonded Gla-sC and the secondary-oxygen-bonded $\mathrm{Glc}$-sO are most stable. We note that there is an agglomeration of elements for the ${ }^{*} \mathrm{C}$ descriptor. For both pairs of descriptors, the map regions are consistent with the actual, directly computed intermediate binding energies, correctly showing the right intermediate for each metal (this is an indicator of the accuracy of the fit). However, with $* \mathrm{C}$ and $* \mathrm{O}$, the energy margins are smaller.

For the second dehydrogenation step (Figure $2 b, 2 d$ ), we see that the maps are qualitatively similar. As a general rule, it can be seen that the thermodynamically most stable intermediates typically go from two carbon bonds through one carbon bond and one oxygen bond to two oxygen bonds as the relative bond strength of the oxygen descriptor is increased. We distinguish between second-dehydrogenation products available by dehydrogenation of G1a-sC and G1c-sO (bound through the secondary group) on one hand, and G1b-pC and G1d-pO (bound through a primary group) on the other, as seen in Figure 1. Since Gla-sC and G1c-sO are most stable, the products originated by dehydrogenating them ( $\mathrm{G} 2 \mathrm{a}-\mathrm{sCsO}$, $\mathrm{G} 2 \mathrm{~b}-\mathrm{pCsC}$, and $\mathrm{G} 2 \mathrm{i}-\mathrm{pO} \mathrm{O} \mathrm{O}$ ) are referred to as direct products below, while the products stemming from G1b-pC and G1dpO (G2d-pCpC and G2j-pOpO) are referred to as indirect products. "Indirect" should be interpreted in the sense that their precursors are not the most thermodynamically favored in the first dehydrogenation step. G1b-pC, G1d-pO, G2d-pCpC, and $\mathrm{G} 2 \mathrm{j}-\mathrm{pOpO}$ could still be produced, however, if the selectivity is less than complete or if the kinetics is more favorable to the dehydrogenation of the primary group.

Starting with the direct products, indicated with solid colors, $\mathrm{G} 2 \mathrm{~b}-\mathrm{pCsC}$ is the preferred intermediate over a wide range from strong to medium bond strength of the carbon descriptor as long as the oxygen descriptor binds somewhat weakly to the 

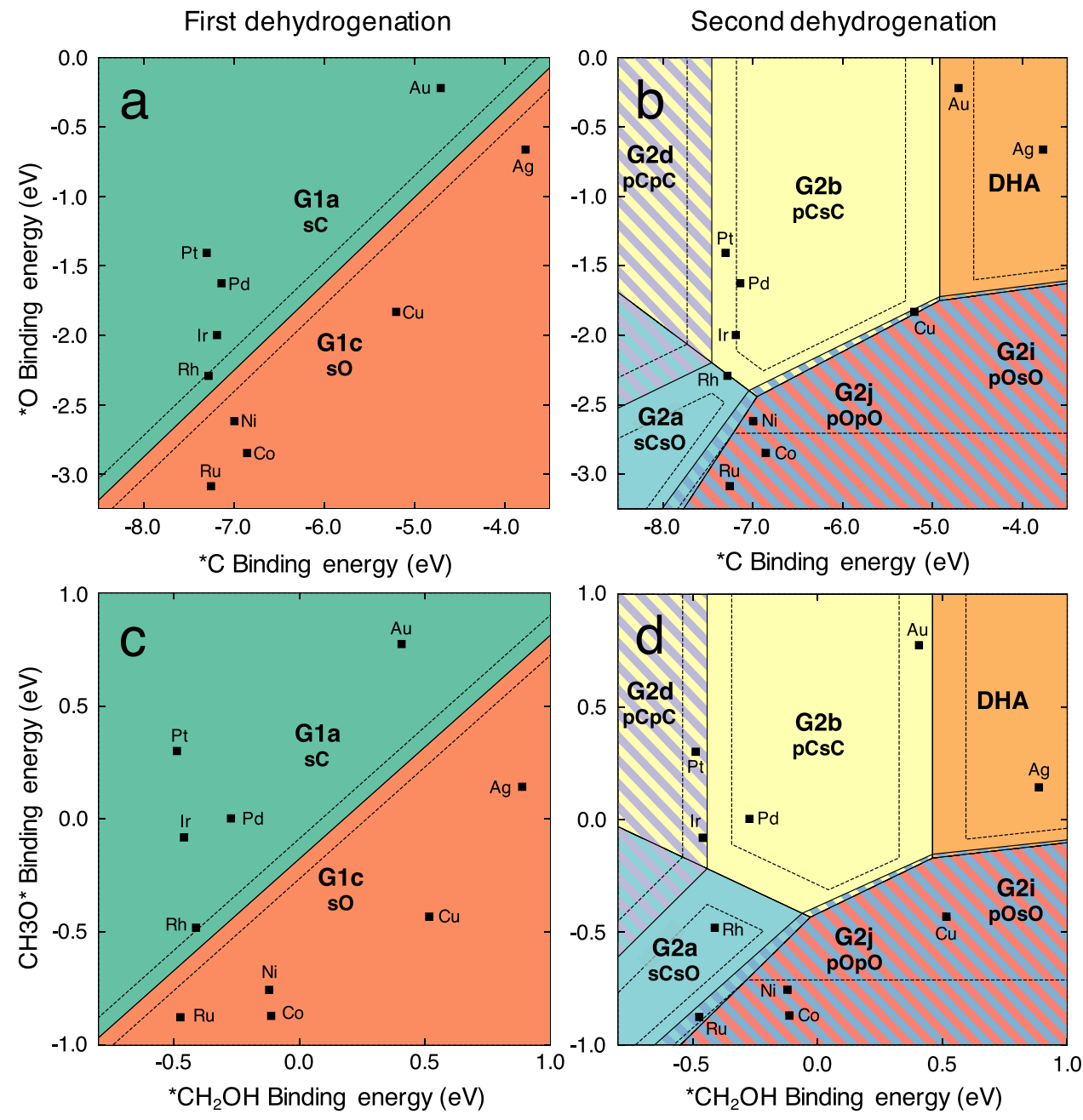

Figure 5. Selectivity for first-dehydrogenated and second-dehydrogenated intermediates of glycerol as a function of the binding energy of $\mathrm{O}^{*}$ and $* \mathrm{C}(\mathrm{a}, \mathrm{b})$ and of $* \mathrm{CH}_{2} \mathrm{OH}$ and $\mathrm{CH}_{3} \mathrm{O} *(\mathrm{c}, \mathrm{d})$. In the striped areas, the indirect products (see the text for definition) G2d (purple) and G2j (blue) are most stable. Dashed lines indicate $0.1 \mathrm{eV}$ margin for the intermediates.

surface. If both descriptors bind weakly, the van der Waalsbound DHA becomes the preferred product. On the other hand, if both descriptors are strongly bound, G2a-sCsO (DHA with broken $\mathrm{C}=\mathrm{O}$ double bond) is preferred, and if the binding energy of carbon is medium to small, the G2i-pOsO instead dominates. If we include indirect products, there are regimes where $\mathrm{G} 2 \mathrm{~d}-\mathrm{pCpC}$ and $\mathrm{G} 2 \mathrm{j}$-pOpO are the most energetically favorable, preferring strong carbon bonding and oxygen bonding, respectively. These are indicated with striped colors.

For the second dehydrogenation, the maps are mostly consistent, such that the descriptor energies on a metal correctly predict the thermodynamically most stable intermediates from direct DFT calculations. For the $* \mathrm{C}$ and $* \mathrm{O}$ in Figure $5 \mathrm{~b}$, the exceptions are (1) iridium, which should favor $\mathrm{G} 2 \mathrm{i}-\mathrm{pO} \mathrm{OO}$ as a direct product and $\mathrm{G} 2 \mathrm{~d}-\mathrm{pCpC}$ as an indirect product; (2) platinum, which should prefer $\mathrm{G} 2 \mathrm{~d}-\mathrm{pCpC}$ as a direct product; and (3) copper, which should form G2i-pOsO as a direct product. For $* \mathrm{CH}_{2} \mathrm{OH}$ and $\mathrm{CH}_{3} \mathrm{O} *$ (Figure $5 \mathrm{~d}$ ), the exceptions are (1) ruthenium, which should exclusively favor G2a-sCsO; (2) iridium, which should prefer $\mathrm{G} 2 \mathrm{a}-\mathrm{sCsO}$ as a direct product (but G2d-pCpC as indirect); and (3) gold, which should form DHA. All deviations are relatively close to the borders, as seen by the $0.1 \mathrm{eV}$ margin lines. Please note that $\mathrm{G} 2 \mathrm{a}-\mathrm{sCsO}$, in particular, is not too well fitted, as seen above. Furthermore, G2i-pOsO and G2j-pOpO have almost the same energies and slopes, which is reflected by the horizontal dashed line in the lower right region, marking the border if the energy of $\mathrm{G} 2 \mathrm{i}$-pOsO shifts $-0.1 \mathrm{eV}$ relative to G2j-pOpO.

So far, we have only discussed thermodynamics, but it is worthwhile to make some remarks on the kinetics. In the presence of water, $\mathrm{O}-\mathrm{H}$ bond breaking is favored over $\mathrm{C}-\mathrm{H}$ bond breaking since water molecules mediate the proton transfer. ${ }^{32,56}$ Even when the reactants are in gas phase, $\mathrm{O}-\mathrm{H}$ bond breaking should be favored over $\mathrm{C}-\mathrm{H}$ bond breaking, relative to other alcohols, because of self-mediation in the form of hydrogen bonding between the three $\mathrm{OH}$ groups. ${ }^{57,58}$ Specifically, on rhodium, the barrier is $0.1 \mathrm{eV}$ smaller for $\mathrm{O}-\mathrm{H}$ vs $\mathrm{C}-\mathrm{H}$ bond breaking in glycerol $(0.67$ vs $0.77 \mathrm{eV})$ even without the presence of water. ${ }^{57}$ On platinum, the $\mathrm{C}-\mathrm{H}$ breaking is slightly favored ( 0.71 vs $0.74 \mathrm{eV})$, while dehydrogenation of the secondary group has a $0.13 \mathrm{eV}$ lower barrier than the primary group. ${ }^{33}$ There are, to our knowledge, no studies on glycerol electrooxidation barriers; the qualitative trends are probably similar to the heterogeneous catalysis case, but the magnitude of the relevant barriers has not been determined. 
As discussed in our previous paper, ${ }^{50}$ the most common glycerol electrooxidation pathway reported in the experimental literature (typically on platinum, palladium, and gold) is through glyceraldehyde (i.e., deprotonation of the primary carbon and oxygen). ${ }^{9}$ This is in contrast to the calculated surface thermodynamics. The possible causes for this are (1) spontaneous deprotonation of a primary $\mathrm{OH}$ group in alkaline solution prior to adsorption; ${ }^{59-61}$ (2) kinetics; (3) steric hindrance; and (4) transformation of the desorbed products in solution, such as Lobry de Bruyn-Alberda van Ekenstein isomerizations between DHA, propene-1,2,3-triol, and glyceraldehyde, ${ }^{62,63}$ since the products are normally detected in solution rather than on the surface. If we also consider nonelectrochemical oxidation in water, gold- and platinum-based catalysts are also selective toward glyceraldehyde and its oxidation products, ${ }^{7}$ which excludes the first cause. It is clear that there remains work to be done to connect the theory and experiment in the field.

\section{CONCLUSIONS}

The pair of descriptors $* \mathrm{C}$ and $* \mathrm{O}$ on one hand and $* \mathrm{CH}_{2} \mathrm{OH}$ and $\mathrm{CH}_{3} \mathrm{O}^{*}$ on the other are used to establish scaling relations over a number of close-packed transition metals $(\mathrm{Ru}, \mathrm{Co}, \mathrm{Rh}$, $\mathrm{Ir}, \mathrm{Ni}, \mathrm{Pd}, \mathrm{Pt}, \mathrm{Cu}, \mathrm{Ag}$, and $\mathrm{Au}$ ) for the first two steps of glycerol dehydrogenation. We find that the latter pair is better at capturing the behavior in terms of errors, distinguishability with regard to descriptor energy, and intuitive slopes. We find that the main reason for the accuracy of $* \mathrm{CH}_{2} \mathrm{OH}$ over $* \mathrm{C}$ is that the glycerol intermediates bound with carbon also interact through the adjacent $* \mathrm{OH}$ group. For the oxygen descriptors, we find that the difference between $\mathrm{CH}_{3} \mathrm{O} *$ and $* \mathrm{O}$ is mostly due to the difference between a single and a double bond, as $* \mathrm{OH}$ is comparable in accuracy to $\mathrm{CH}_{3} \mathrm{O}^{*}$. Exceptions to this trend are the van der Waals-bound glycerol and dihydroxyacetone, which scale well with $* \mathrm{C}$ although similar absolute errors are observed with $* \mathrm{O}$.

Using the scaling relations, we find that the first dehydrogenation is selective to oxidation of the carbon or oxygen of the secondary group, depending on the relative binding energy of the descriptors. Continuing from these intermediates in a second dehydrogenation step, we find that the selectivity is steered toward four different intermediates. These are (1) G2b-pCsC, which prefers weak binding energy of the oxygen descriptor and medium to strong binding energy of the carbon descriptor; (2) DHA, which prefers weak binding energy of both descriptors; (3) G2a-sCsO, which prefers strong binding energy of both descriptors; and (4) G2ipOsO, which prefers stronger binding of the oxygen descriptor than the carbon descriptor. If we consider second-step intermediates stemming from oxidation of the primary group, also G2d-pCpC and G2j-pOpO are most stable in some regions.

The simple scaling relations with $* \mathrm{CH}_{2} \mathrm{OH}$ and $\mathrm{CH}_{3} \mathrm{O} *$ can be used to screen for catalyst formulations for glycerol valorization by dehydrogenation. Building on the work in our previous paper, ${ }^{50}$ the selectivity for products such as DHA, GLYD, and HYDP can be deduced from the two first steps of glycerol dehydrogenation. The pathways of continued dehydrogenation on the surface can be studied by establishing new scaling relations to further intermediates that are similar to these two descriptors.

The present work also gives important insight into the nature of any alcohol intermediates bound through carbon with a hydroxyl group, as the negative dependence on $* \mathrm{OH}$ should be common. The alternative to calculating the ${ }^{*} \mathrm{CH}_{2} \mathrm{OH}$ (and $\mathrm{CH}_{3} \mathrm{O} *$ ) binding energies on a number of surfaces is to use the existing databases of $* \mathrm{C}, * \mathrm{O}, * \mathrm{OH}$, etc. It is then necessary to expect the oxygen dependence and analyze it through multilinear regression as carried out in the Supporting Information.

\section{ASSOCIATED CONTENT}

\section{Supporting Information}

The Supporting Information is available free of charge at https://pubs.acs.org/doi/10.1021/acscatal.0c04186.

Selectivity of the first two glycerol dehydrogenation steps determined using scaling relationships, containing DFT energies, Bader charges, tabulated scaling relations, and selectivity maps (PDF)

Structure and data files (ZIP)

\section{AUTHOR INFORMATION}

\section{Corresponding Author}

Mikael Valter - Department of Physics, Chalmers University of Technology, SE-412 96 Göteborg, Sweden; 다이.org/ 0000-0002-7718-3525; Phone: +46 3177256 11; Email: mikael.valter@fysik.su.se

\section{Authors}

Egon Campos dos Santos - Department of Physics, Stockholm University, SE-106 91 Stockholm, Sweden

Lars G. M. Pettersson - Department of Physics, Stockholm University, SE-106 91 Stockholm, Sweden

Anders Hellman - Department of Physics and the Competence Centre for Catalysis, Chalmers University of Technology, SE41296 Göteborg, Sweden; O orcid.org/0000-0002-1821$159 \mathrm{X}$

Complete contact information is available at:

https://pubs.acs.org/10.1021/acscatal.0c04186

\section{Notes}

The authors declare no competing financial interest.

\section{ACKNOWLEDGMENTS}

M.V. and A.H. gratefully acknowledge the support from Formas and the Swedish Research Council. E.C.d.S. and L.G.M.P. were supported by the Swedish Foundation for Strategic Research (SSF) through grant number EM16-0010. The electronic structure calculations were performed using resources provided by the Swedish National Infrastructure for Computing (SNIC) at the NSC and UPPMAX highperformance computer centers.

\section{REFERENCES}

(1) Gerpen, J. V. Biodiesel Processing and Production. Fuel Process. Technol. 2005, 86, 1097-1107.

(2) Werpy, T. A.; Holladay, J. E.; White, J. F. Top Value Added Chemicals from Biomass: I Results of Screening for Potential Candidates from Sugars and Synthesis Gas, 2004.

(3) Kirubakaran, M.; Selvan, V. A. M. A Comprehensive Review of Low Cost Biodiesel Production from Waste Chicken Fat. Renewable Sustainable Energy Rev. 2018, 82, 390-401.

(4) Gu, Y.; Jérôme, F. Glycerol as a Sustainable Solvent for Green Chemistry. Green Chem. 2010, 12, 1127-1138. 
(5) Behr, A.; Eilting, J.; Irawadi, K.; Leschinski, J.; Lindner, F. Improved Utilisation of Renewable Resources: New Important Derivatives of Glycerol. Green Chem. 2008, 10, 13-30.

(6) Zhang, H.; Xu, C. C.; Zhou, K.; Yang, S. Chemo-catalytic Esterification and Transesterification over Organic Polymer-Based Catalysts for Biodiesel Synthesis. Curr. Org. Chem. 2019, 23, 21902203.

(7) Pagliaro, M.; Ciriminna, R.; Kimura, H.; Rossi, M.; Della Pina, C. From Glycerol to Value-Added Products. Angew. Chem., Int. Ed. 2007, 46, 4434-4440.

(8) Amaniampong, P. N.; Trinh, Q. T.; Varghese, J. J.; Behling, R.; Valange, S.; Mushrif, S. H.; Jérôme, F. Unraveling the Mechanism of the Oxidation of Glycerol to Dicarboxylic Acids over a Sonochemically Synthesized Copper Oxide Catalyst. Green Chem. 2018, 20, 2730-2741.

(9) Houache, M. S. E.; Hughes, K.; A. Baranova, E. Study on Catalyst Selection for Electrochemical Valorization of Glycerol. Sustainable Energy Fuels 2019, 3, 1892-1915.

(10) Simões, M.; Baranton, S.; Coutanceau, C. Electrochemical Valorisation of Glycerol. ChemSusChem 2012, 5, 2106-2124.

(11) Morfeldt, J.; Nijs, W.; Silveira, S. The Impact of Climate Targets on Future Steel Production -an Analysis Based on a Global Energy System Model. J. Cleaner Prod. 2015, 103, 469-482.

(12) Karakaya, E.; Nuur, C.; Assbring, L. Potential Transitions in the Iron and Steel Industry in Sweden: Towards a Hydrogen-Based Future. J. Cleaner Prod. 2018, 195, 651-663.

(13) Pagliaro, M. V.; Bellini, M.; Bevilacqua, M.; Filippi, J.; Folliero, M. G.; Marchionni, A.; Miller, H. A.; Oberhauser, W.; Caporali, S.; Innocenti, M.; Vizza, F. Carbon Supported Rh Nanoparticles for the Production of Hydrogen and Chemicals by the Electroreforming of Biomass-Derived Alcohols. RSC Adv. 2017, 7, 13971-13978.

(14) Chen, Y. X.; Lavacchi, A.; Miller, H. A.; Bevilacqua, M.; Filippi, J.; Innocenti, M.; Marchionni, A.; Oberhauser, W.; Wang, L.; Vizza, F. Nanotechnology Makes Biomass Electrolysis More Energy Efficient than Water Electrolysis. Nat. Commun. 2014, 5, No. 4036.

(15) Coutanceau, C.; Baranton, S. Electrochemical Conversion of Alcohols for Hydrogen Production: A Short Overview. Wiley Interdiscip. Rev.: Energy Environ. 2016, 5, 388-400.

(16) Liu, B.; Gao, F. Navigating Glycerol Conversion Roadmap and Heterogeneous Catalyst Selection Aided by Density Functional Theory: A Review. Catalysts 2018, 8, No. 44.

(17) Talebian-Kiakalaieh, A.; Amin, N. A. S.; Rajaei, K.; Tarighi, S. Oxidation of Bio-Renewable Glycerol to Value-Added Chemicals through Catalytic and Electro-Chemical Processes. Appl. Energy 2018, 230, 1347-1379.

(18) BASF, Tradition of Ideas: Formic Acid, (accessed Mar 12, 2020).

(19) Bagheri, S.; Julkapli, N. M.; Yehye, W. A. Catalytic Conversion of Biodiesel Derived Raw Glycerol to Value Added Products. Renewable Sustainable Energy Rev. 2015, 41, 113-127.

(20) Dodekatos, G.; Schünemann, S.; Tüysüz, H. Recent Advances in Thermo-, Photo-, and Electrocatalytic Glycerol Oxidation. ACS Catal. 2018, 8, 6301-6333.

(21) Johnson, D. T.; Taconi, K. A. The Glycerin Glut: Options for the Value-Added Conversion of Crude Glycerol Resulting from Biodiesel Production. Environ. Prog. 2007, 26, 338-348.

(22) Abild-Pedersen, F.; Greeley, J.; Studt, F.; Rossmeisl, J.; Munter, T. R.; Moses, P. G.; Skúlason, E.; Bligaard, T.; Nørskov, J. K. Scaling Properties of Adsorption Energies for Hydrogen-Containing Molecules on Transition-Metal Surfaces. Phys. Rev. Lett. 2007, 99, No. 016105.

(23) Calle-Vallejo, F.; Loffreda, D.; Koper, M. T. M.; Sautet, P. Introducing Structural Sensitivity into Adsorption-Energy Scaling Relations by Means of Coordination Numbers. Nat. Chem. 2015, 7, 403-410.

(24) Wang, S.; et al. Universal Transition State Scaling Relations for (De)hydrogenation over Transition Metals. Phys. Chem. Chem. Phys. 2011, 13, 20760-20765.

(25) Wang, S.; Temel, B.; Shen, J.; Jones, G.; Grabow, L. C.; Studt, F.; Bligaard, T.; Abild-Pedersen, F.; Christensen, C. H.; Nørskov, J. K.
Universal Brønsted-Evans-Polanyi Relations for C-C, C-O, C-N, N-O, N-N, and O-O Dissociation Reactions. Catal. Lett. 2011, 141, 370373.

(26) Bligaard, T.; Nørskov, J. K.; Dahl, S.; Matthiesen, J.; Christensen, C. H.; Sehested, J. The Brønsted-Evans-Polanyi Relation and the Volcano Curve in Heterogeneous Catalysis. J. Catal. 2004, 224, 206-217.

(27) Cheng, J.; Hu, P.; Ellis, P.; French, S.; Kelly, G.; Lok, C. M. Brønsted-Evans-Polanyi Relation of Multistep Reactions and Volcano Curve in Heterogeneous Catalysis. J. Phys. Chem. C 2008, 112, 13081311.

(28) Nørskov, J. K.; Bligaard, T.; Rossmeisl, J.; Christensen, C. H. Towards the computational design of solid catalysts. Nat. Chem. 2009, $1,37-46$

(29) Seh, Z. W.; Kibsgaard, J.; Dickens, C. F.; Chorkendorff, I.; Nørskov, J. K.; Jaramillo, T. F. Combining Theory and Experiment in Electrocatalysis: Insights into Materials Design. Science 2017, 355, No. eaad4998.

(30) Montemore, M. M.; Medlin, J. W. A Unified Picture of Adsorption on Transition Metals through Different Atoms. J. Am. Chem. Soc. 2014, 136, 9272-9275.

(31) Greeley, J. Theoretical Heterogeneous Catalysis: Scaling Relationships and Computational Catalyst Design. Annu. Rev. Chem. Biomol. Eng. 2016, 7, 605-635.

(32) Zaffran, J.; Michel, C.; Delbecq, F.; Sautet, P. Towards More Accurate Prediction of Activation Energies for Polyalcohol Dehydrogenation on Transition Metal Catalysts in Water. Catal. Sci. Technol. 2016, 6, 6615-6624.

(33) Liu, B.; Greeley, J. A Density Functional Theory Analysis of Trends in Glycerol Decomposition on Close-Packed Transition Metal Surfaces. Phys. Chem. Chem. Phys. 2013, 15, 6475-6485.

(34) Kresse, G.; Hafner, J. Ab Initio Molecular Dynamics for Liquid Metals. Phys. Rev. B: Condens. Matter Mater. Phys. 1993, 47, 558-561.

(35) Kresse, G.; Furthmüller, J. Efficiency of Ab-Initio Total Energy Calculations for Metals and Semiconductors Using a Plane-Wave Basis Set. Comput. Mater. Sci. 1996, 6, 15-50.

(36) Kresse, G.; Furthmüller, J. Efficient Iterative Schemes for Ab Initio Total-Energy Calculations Using a Plane-Wave Basis Set. Phys. Rev. B: Condens. Matter Mater. Phys. 1996, 54, 11169-11186.

(37) Blöchl, P. E. Projector Augmented-Wave Method. Phys. Rev. B: Condens. Matter Mater. Phys. 1994, 50, 17953-17979.

(38) Klimeš, J.; Bowler, D. R.; Michaelides, A. Chemical Accuracy for the van Der Waals Density Functional. J. Phys.: Condens. Matter 2010, 22, No. 022201.

(39) Klimeš, J.; Bowler, D. R.; Michaelides, A. Van Der Waals Density Functionals Applied to Solids. Phys. Rev. B: Condens. Matter Mater. Phys. 2011, 83, No. 195131.

(40) Becke, A. D. On the Large-Gradient Behavior of the Density Functional Exchange Energy. J. Chem. Phys. 1986, 85, 7184-7187.

(41) Björk, J.; Stafström, S. Adsorption of Large Hydrocarbons on Coinage Metals: A van der Waals Density Functional Study. ChemPhysChem 2014, 15, 2851-2858.

(42) Lozano, A.; Escribano, B.; Akhmatskaya, E.; Carrasco, J. Assessment of van der Waals inclusive density functional theory methods for layered electroactive materials. Phys. Chem. Chem. Phys. 2017, 19, 10133-10139.

(43) Baltrusaitis, J.; Valter, M.; Hellman, A. Geometry and Electronic Properties of Glycerol Adsorbed on Bare and TransitionMetal Surface-Alloyed $\mathrm{Au}(111)$ : A Density Functional Theory Study. J. Phys. Chem. C 2016, 120, 1749-1757.

(44) Wellendorff, J.; Lundgaard, K. T.; Møgelhøj, A.; Petzold, V.; Landis, D. D.; Nørskov, J. K.; Bligaard, T.; Jacobsen, K. W. Density functionals for surface science: Exchange-correlation model development with Bayesian error estimation. Phys. Rev. B: Condens. Matter Mater. Phys. 2012, 85, No. 235149.

(45) Wellendorff, J.; Silbaugh, T. L.; Garcia-Pintos, D.; Nørskov, J. K.; Bligaard, T.; Studt, F.; Campbell, C. T. A Benchmark Database for Adsorption Bond Energies to Transition Metal Surfaces and 
Comparison to Selected DFT Dunctionals. Surf. Sci. 2015, 640, 3644.

(46) Gautier, S.; N. Steinmann, S.; Michel, C.; Fleurat-Lessard, P.; Sautet, P. Molecular Adsorption at $\mathrm{Pt}(111)$. How Accurate Are DFT Functionals. Phys. Chem. Chem. Phys. 2015, 17, 28921-28930.

(47) Perdew, J. P.; Burke, K.; Ernzerhof, M. Generalized Gradient Approximation Made Simple. Phys. Rev. Lett. 1996, 77, 3865.

(48) Monkhorst, H. J.; Pack, J. D. Special Points for Brillouin-Zone Integrations. Phys. Rev. B: Condens. Matter Mater. Phys. 1976, 13, $5188-5192$.

(49) Zaffran, J.; Michel, C.; Delbecq, F.; Sautet, P. Trade-off Between Accuracy and Universality in Linear Energy Relations for Alcohol Dehydrogenation on Transition Metals. J. Phys. Chem. C 2015, 119, 12988-12998.

(50) Valter, M.; Dos Santos, E. C.; Pettersson, L. G.; Hellman, A. Partial Electrooxidation of Glycerol on Close-Packed Transition Metal Surfaces: Insights from First-Principles Calculations. J. Phys. Chem. C 2020, 124, 17907-17915.

(51) Jones, G.; Studt, F.; Abild-Pedersen, F.; Nørskov, J. K.; Bligaard, T. Scaling Relationships for Adsorption Energies of C2 Hydrocarbons on Transition Metal Surfaces. Chem. Eng. Sci. 2011, 66, 6318-6323.

(52) Denisova, A. Hyperconjugation in Group 14 Organic Compounds: Design and Property Investigations, Ph.D. Thesis; Acta Universitatis Upsaliensis, 2017.

(53) Öström, H.; Ogasawara, H.; Näslund, L.-Å.; Pettersson, L.; Nilsson, A. Physisorption-Induced $\mathrm{CH}$ bond Elongation in Methane. Phys. Rev. Lett. 2006, 96, No. 146104.

(54) Schiros, T.; Ogasawara, H.; Näslund, L.-Å.; Andersson, K. J.; Ren, J.; Meng, S.; Karlberg, G.; Odelius, M.; Nilsson, A.; Pettersson, L. G. Cooperativity in Surface Bonding and Hydrogen Bonding of Water and Hydroxyl at Metal Surfaces. J. Phys. Chem. C 2010, 114, 10240-10248.

(55) Mamun, O.; Winther, K. T.; Boes, J. R.; Bligaard, T. HighThroughput Calculations of Catalytic Properties of Bimetallic Alloy Surfaces. Sci. Data 2019, 6, No. 76.

(56) Michel, C.; Zaffran, J.; M. Ruppert, A.; Matras-Michalska, J.; Jȩdrzejczyk, M.; Grams, J.; Sautet, P. Role of Water In Metal Catalyst Performance for Ketone Hydrogenation: a Joint Experimental and Theoretical Study on Levulinic Acid Conversion into GammaValerolactone. Chem. Commun. 2014, 50, 12450-12453.

(57) Zaffran, J.; Michel, C.; Auneau, F.; Delbecq, F.; Sautet, P. Linear Energy Relations as Predictive Tools for Polyalcohol Catalytic Reactivity. ACS Catal. 2014, 4, 464-468.

(58) Michel, C.; Auneau, F.; Delbecq, F.; Sautet, P. C-H versus O-H Bond Dissociation for Alcohols on a $\mathrm{Rh}(111)$ Surface: A Strong Assistance from Hydrogen Bonded Neighbors. ACS Catal. 2011, 1, $1430-1440$.

(59) Kwon, Y.; Lai, S. C. S.; Rodriguez, P.; Koper, M. T. M. Electrocatalytic Oxidation of Alcohols on Gold in Alkaline Media: Base or Gold Catalysis. J. Am. Chem. Soc. 2011, 133, 6914-6917.

(60) Suzuki, N. Y.; Santiago, P. V. B.; Galhardo, T. S.; Carvalho, W. A.; Souza-Garcia, J.; Angelucci, C. A. Insights of Glycerol Electrooxidation on Polycrystalline Silver Electrode. J. Electroanal. Chem. 2016, 780, 391-395.

(61) Sandrini, R. M. L. M.; Sempionatto, J. R.; Herrero, E.; Feliu, J. M.; Souza-Garcia, J.; Angelucci, C. A. Mechanistic Aspects of Glycerol Electrooxidation on $\mathrm{Pt}(111)$ Electrode in Alkaline Media. Electrochem. Commun. 2018, 86, 149-152.

(62) Angyal, S. J. Glycoscience: Epimerisation, Isomerisation and Rearrangement Reactions of Carbohydrates. In Topics in Current Chemistry, Stütz, A. E., Ed.; Springer: Berlin, Heidelberg, 2001; pp 114.

(63) Yaylayan, V. A.; Harty-Majors, S.; Ismail, A. A. Investigation of Dl-Glyceraldehyde-Dihydroxyacetone Interconversion by FTIR Spectroscopy. Carbohydr. Res. 1999, 318, 20-25. 